Article

\title{
Evaluating Inhibition of the Epidermal Growth Factor (EGF)-Induced Response of Mutant MCF10A Cells with an Acoustic Sensor
}

\author{
Marcela P. Garcia ${ }^{1}$, Ammar Shahid ${ }^{2}$, Jennifer Y. Chen ${ }^{1}$ and Jun Xi ${ }^{1, *}$ \\ 1 Department of Chemistry, Drexel University, 3141 Chestnut Street, Philadelphia, PA 19104, USA; \\ E-Mails: mpg36@drexel.edu (M.P.G.); jyc29@drexel.edu (J.Y.C.) \\ 2 Department of Biology, Drexel University, 3141 Chestnut Street, Philadelphia, PA 19104, USA; \\ E-Mail: as954@drexel.edu
}

* Author to whom correspondence should be addressed; E-Mail: jx35@drexel.edu; Tel.: +1-215-895-2648; Fax: +1-215-895-1265.

Received: 29 August 2012; in revised form: 8 October 2012 / Accepted: 7 November 2012 / Published: 13 November 2012

\begin{abstract}
Many cancer treatments rely on inhibition of epidermal growth factor (EGF)-induced cellular responses. Evaluating drug effects on such responses becomes critical to the development of new cancer therapeutics. In this report, we have employed a label-free acoustic sensor, the quartz crystal microbalance with dissipation monitoring (QCM-D), to track the EGF-induced response of mutant MCF10A cells under various inhibitory conditions. We have identified a complex cell de-adhesion process, which can be distinctly altered by inhibitors of signaling pathways and cytoskeleton formation in a dose-dependent manner. The dose dependencies of the inhibitors provide $\mathrm{IC}_{50}$ values which are in strong agreement with the values reported in the literature, demonstrating the sensitivity and reliability of the QCM-D as a screening tool. Using immunofluorescence imaging, we have also verified the quantitative relationship between the $\Delta D$-response (change in energy dissipation factor) and the level of focal adhesions quantified with the areal density of immunostained vinculin under those inhibitory conditions. Such a correlation suggests that the dynamic restructuring of focal adhesions can be assessed based on the time-dependent change in $\Delta D$-response. Overall, this report has shown that the QCM-D has the potential to become an effective sensing platform for screening therapeutic agents that target signaling and cytoskeletal proteins.
\end{abstract}


Keywords: QCM-D; biosensors; label free; EGFR; cell adhesion; cell signaling; cytoskeleton; inhibition; cancer development; drug screening

\section{Introduction}

It is well known that the epidermal growth factor receptor (EGFR) regulates cell growth, proliferation, motility and differentiation through its downstream signaling pathways [1,2]. These downstream pathways such as the mitogen-activated protein kinase/extracellular signal-regulated kinase (MAPK/ERK) pathway [3], the phosphoinositide 3-kinase (PI3K) pathway [4], and the phospholipase C (PLC) pathway [5], are activated when epidermal growth factor (EGF) binds to the extracellular domain of EGFR. An abnormality in the EGFR system, such as overexpression and/or mutation of EGFR, increased production of the ligands (e.g., EGF) of EGFR, or downregulation of EGFR, can interfere with the tight regulation of these signaling pathways [6] and lead to the development of epithelial malignancies in humans such as cancers [7]. A better understanding of the effects of abnormalities in the EGFR system on cells will contribute to the understanding of the role of these abnormalities in cancer development, which, in turn, can lead ultimately to more effective cancer diagnosis, treatment, and prognosis [8].

Conventional approaches to the study of cellular responses to EGFR signaling rely on high spatial resolutions of radioactive or fluorescent labels to track the location, trafficking, and organization of one or more types of signaling molecules in a specific pathway. Such approaches have been successful in identification of some of the main activators, effectors, enzymes, and substrates in EGFR signaling [9]. However, because of the complexity of the signaling network and intracellular dynamics [10,11], information on specific components of a signaling pathway usually fails to provide the functional response of the whole cell. In addition, the temporal resolution of these label-based approaches is not high, thereby limiting the amount of information that can be obtained on the dynamics of cell signaling. Incorporation of various labels may also create a non-native and physiologically irrelevant cellular environment, which could potentially lead to ambiguous results [12-14].

These problems can be circumvented with the use of label-free biosensor technologies, which are capable of providing integrated and phenotypic readouts at the whole cell level [15]. Compared with label-based technologies, label-free biosensor technologies based on either optical or non-optical sensing platforms [16,17], are, for the most part, non-invasive, and capable of real-time measurement of cell signaling kinetics with high temporal resolution and high sensitivity [18]. The primary examples of optical, label-free sensing platforms are resonant waveguide grating (RWG) [19] and surface plasmon resonance (SPR) [20], both of which use a surface bound evanescent wave to determine the refractive indices of biomolecules [21]. Meanwhile, electrical impedance, acoustic resonance, microcantilevers, field effect nanowires and differential calorimetry belong in the category of non-optical, label-free biosensors [16]. The benchmark technology among these non-optical platforms is electrical impedance, which uses its sensitivity to ionic movement under electric fields to indicate morphological changes in a layer of cells [22]. 
Over the years, the applications of label-free biosensors in cell biology, including aspects of cell adhesion, cell barrier functions, cell signaling, and viral infection, have been steadily increasing [15]. In particular, the detection of functional responses of cells to EGFR signaling has attracted tremendous interests due to the biological significance of EGFR in cancer diagnosis, treatment, and prognosis. RWG, SPR, electrical impedance, and acoustic resonnance-based detection methods for this application have all been reported [23-26].

The quartz crystal microbalance with dissipation monitoring (QCM-D) is an acoustic resonance-based sensor that measures the changes in mass (from the change in vibrational frequency $\Delta f$ ) and mechanical properties (from the change in energy dissipation factor $\Delta D$ ) of a layer of biomolecules attached to the surface of an oscillating AT-cut quartz crystal [27]. The correlation between $\Delta D$ and $\Delta f$ can be used as an indicator of the cell-substrate interaction [28]. Like other label-free biosensors, the QCM-D is non-invasive and highly sensitive, and has a unique capability to simultaneously assess changes in mass and energy dissipation of the material that is coupled to the surface of the sensor crystal. This capability makes it a useful tool in the field of material and biological sciences [29,30].

In recent years, the QCM-D has become particularly attractive in the field of cell biology for its ability to monitor the interaction between cells and the surface to which they are attached [31-36] and determine the kinetics of cell attachment and spreading [27,37,38]. Applications of the QCM-D to the study of functional response of cells to receptor-mediated cell signaling have also begun to emerge as a result of our own research efforts $[23,39,40]$. We have successfully measured the short-term responses of human epidermoid carcinoma A431 cells to EGFR-mediated signaling based on real-time monitoring of changes in the dissipation factor $(\Delta D)$ and frequency responses $(\Delta f)$ [23]. We have also demonstrated the capability of the QCM-D in tracking the functional responses of both wildtype and mutant MCF10A cells during the EGF-induced cell de-adhesion (i.e., the reverse process of adhesion) $[39,41]$. A correlation between the time-dependent $\Delta D$-response of the QCM-D and the level of focal adhesions of the cells has been established in those studies [39]. Based on this strong correlation, we have examined the regulation of this dynamic de-adhesion by the downstream pathways of EGFR signaling including the PI3K, MAPK/ERK, and PLC pathways [39].

The present paper reports an investigation of the EGF-induced de-adhesion of mutant MCF10A cells from the surface to which they are attached. Compared with wildtype MCF10A cells, these mutant cells possess a much higher level of EGFR [42], a situation that mimics the abnormal levels of EGFR in many types of solid tumors [43]. Such an abnormality has been linked to dysregulated cellular functions, including cell adhesion, which is part of pathological processes $[44,45]$ that can promote tumor invasion and metastasis $[6,46]$. Finding an effective way to suppress tumor migration by limiting the EGF-induced cell de-adhesion might contribute to the development of a potentially more effective cancer treatment. Thus, the focus of this study was on evaluation of the inhibition of the EGF-induced de-adhesion in mutant MCF10A cells. The QCM-D was used to track the real-time responses of cells during this dynamic process. This study shows that the EGF-induced cell de-adhesion can be distinctly altered by inhibitors of signaling pathways (PD158780 for the EGFR activation, LY294002 for the PI3K pathway, U73122 for the PLC pathway, and L-779450 for the MAPK/ERK pathway) and cytoskeleton formation (cytochalasin $\mathrm{D}$ for actin polymerization) in a dose-dependent manner. The potency of each inhibitor as determined by means of the QCM-D corresponds well with the value reported in the literature. This strong agreement strongly suggests that the QCM-D has the 
potential to become an effective sensing platform for screening therapeutic agents that target signaling and cytoskeletal proteins.

\section{Experimental Section}

\subsection{Reagents}

Dulbecco's modified Eagle's medium and Ham's F-12 nutrient mixture (DMEM/F12), horse serum, antibiotics, trypsin-EDTA, HEPES buffer, and HBSS buffer were purchased from Invitrogen. Human epidermal growth factor was purchased from Peprotech. Hydrocortisone, cholera toxin, and insulin were obtained from Sigma-Aldrich. PD158780 was purchased from EMD Bioscience. LY294002 and U73122 were purchased from Cayman Chemical Company, cytochalasin D was purchased from Enzo Life Sciences, and L-779450 (RafKinase Inhibitor IV) was purchased from Calbiochem.

\subsection{Cell Culture}

Mutant MCF-10A cells that overexpress EGFR were derived from stable transfection [42]. They were grown in T75 Corning flasks and maintained under a humidified atmosphere at $37{ }^{\circ} \mathrm{C}$ and $5 \%$ $\mathrm{CO}_{2}$ in DMEM/F12 medium containing $5 \%$ horse serum, $20 \mathrm{ng} / \mathrm{mL}$ EGF, $0.5 \mu \mathrm{g} / \mathrm{mL}$ hydrocortisone, $50 \mathrm{ng} / \mathrm{mL}$ cholera toxin, $10 \mu \mathrm{g} / \mathrm{mL}$ insulin, $100 \mathrm{IU} / \mathrm{mL}$ penicillin, and $100 \mu \mathrm{g} / \mathrm{mL}$ streptomycin. The cells were harvested at $95 \%$ confluency with the treatment of $0.25 \%$ trypsin-EGTA at $37{ }^{\circ} \mathrm{C}$ for $10 \mathrm{~min}$. The cell medium containing trypsin was then removed and the cells were re-suspended in growth medium for plating on sensors and/or coverslips. The maximum number of cell passage used for this study was 12 . The Stratagene mycoplasma plus PCR primer set was used to detect mycoplasma contamination.

\subsection{QCM-D Measurements}

A QCM-D (E4, Q-Sense) was used to record changes in energy dissipation factor $(\Delta D)$ as a function of time at the odd overtone $(n=3)$ as previously described [39]. The sensors were prepared as follows: The QCM-D sensor crystals (gold-surfaced) were first washed with water and ethanol, then were dried under flowing nitrogen gas, and finally were exposed to UV-ozone for $20 \mathrm{~min}$. The sensors were then stored in the tissue culture hood under UV-light for an additional $30 \mathrm{~min}$. The virtually identical behavior of MCF10A cells on both gold-surfaced and glass-surfaced crystals [39] allowed us to proceed to compare the $\Delta D$ responses of the cells on gold-surfaced crystals with the fluorescence images of the cells on glass-surfaced coverslips. The sensors were then placed in a 12-well tissue culture plate along with mutant MCF-10A cells that had been harvested from the T75 culture flask. The cells were allowed to grow on the sensors under a humidified atmosphere at $37{ }^{\circ} \mathrm{C}$ and $5 \% \mathrm{CO}_{2}$.

When they reached $95 \%$ confluency, the cells were starved in serum free medium for $18 \mathrm{~h}$. Each sensor crystal with a cell layer was then mounted in an open module (Q-sense) and incubated in $400 \mu \mathrm{L}$ of the assay buffer (20 mM HEPES in HBSS buffer, $\mathrm{pH} 7.2$ ) at $37{ }^{\circ} \mathrm{C}$. After the stable baselines were established for all four sensors, the cells were then incubated in $400 \mu \mathrm{L}$ of the inhibitor solution at $37{ }^{\circ} \mathrm{C}$ for $40 \mathrm{~min}$. Then the inhibitor solution was replaced with the same volume of $10 \mathrm{nM}$ EGF in the assay buffer containing the same concentration of the inhibitor and the cells were incubated for $3 \mathrm{~h}$. 


\subsection{Fluorescence Imaging}

Cells were seeded on coverslips and allowed to grow to 95\% confluency in a humidified atmosphere at $37{ }^{\circ} \mathrm{C}$ and $5 \% \mathrm{CO}_{2}$. The cells were then starved in serum-free medium for $18 \mathrm{~h}$. Prior to immunostaining, the cells on coverslips were incubated in $1 \mathrm{~mL}$ of the assay buffer at $37{ }^{\circ} \mathrm{C}$ for $1 \mathrm{~h}$, then in the inhibitor solution for $40 \mathrm{~min}$, and finally in $1 \mathrm{~mL}$ of 10-nM EGF solution containing the same concentration of the inhibitor for $3 \mathrm{~h}$. For immunostaining, the cells were fixed in a solution of $0.1 \%$ Triton X-100 and 3\% paraformaldehyde in PHEM buffer (60 mM PIPES, $25 \mathrm{mM}$ HEPES, $10 \mathrm{mM}$ EGTA, $2 \mathrm{mM} \mathrm{MgCl} 2$ and $\mathrm{pH}$ 6.9) at room temperature for $20 \mathrm{~min}$. The fixed cells were first treated with monoclonal mouse anti-vinculin antibody (Invitrogen) at a concentration of 1:200 in blocking buffer (PBS with 2\% BSA) at room temperature for $120 \mathrm{~min}$, and then with Alexafluor 546 goat anti-mouse (Invitrogen) antibody at a concentration of 1:200 in staining buffer (PBS with $2 \% \mathrm{BSA}$ ) at room temperature for $60 \mathrm{~min}$. The coverslips with stained cells were mounted in Vectashield medium DAPI (Vector Laboratories, Inc.) and were imaged with an inverted fluorescence microscope (Zeiss Axioplan 2). All images were processed with the use of Slidebook 5.0 software (Intelligent Imaging Innovations).

\subsection{Data Analysis}

The dose-response curve was created by plotting the average amplitudes $( \pm 1$ std. dev. $)$ of the $\Delta D$-responses versus inhibitor concentrations. Each amplitude value, defined as the absolute value of the difference between the experimental value and the control value, was taken at 40 min of the $\Delta D$-response. $\mathrm{IC}_{50}$ value was calculated with the use of PSI-Plot (Poly Software International) by fitting the data to the following equation:

$$
\Delta D=\frac{a x}{I C_{50}+x}
$$

where $x$ is the concentration of the inhibitor. $a$ corresponds to the maximum $\Delta D$-response, which can be determined through curve fitting. The use of the log functional plot or sigmoid plot for this analysis would not significantly alter the resulting $\mathrm{IC}_{50}$ values.

\subsection{Fluorescence Quantitation}

The areal density of focal adhesions was quantified with ImageJ software (http://rsb.infor.nih.gov/ij/). For each image, the background intensity was first determined from the fluorescence of a selected empty area. Then the density of focal adhesions of a selected cell was obtained by subtracting the background intensity from the total fluorescence intensity (IntDen) of immunostained vinculin. Ten randomly selected cells were analyzed for each image and the standard error of the mean (SEM) was computed. The student t-test was used for statistical analysis of the data set. 


\section{Results and Discussion}

\subsection{Overview}

In our previous study, we had been able to employ the QCM-D to track the EGF-induced cellular responses of confluent monolayers of human epithelial breast MCF-10A cells. The time-dependent $\triangle D$-responses measured by the QCM-D were found to be correlated with dynamic changes in areal density of focal adhesions of the cells [39]. Since the areal density of focal adhesions is quantitatively related to the strength of cell adhesion [47-50], we then used the $\Delta D$-response as an indicator of the strength of cell-substrate adhesion to assess the EGF-induced cell de-adhesion in wildtype MCF10A cells [39]. The cells exhibit a complex de-adhesion process, consisting of de-adhesion, transition, and re-adhesion[39]. In these studies, as in the present study, we used various inhibitors to modulate the EGF-induced $\Delta D$-responses in order to gain mechanistic insights into the regulation of cellular responses.

In the present study, we sought to assess the potential of using the QCM-D as a sensing platform for drug screening. Due to the limited throughput of the QCM-D, where only four independent experiments can be run simultaneously, the QCM-D is far from ready for drug screening. Therefore, the focus of the present study was on sensitivity and reliability. We first evaluated the dose response for inhibition of the EGF-induced cell de-adhesion process in mutant MCF10A cells that overexpress EGFR. We then verified the time dependence of the inhibition with immunofluorescence imaging. Finally, we determined the potency of each inhibitor and compared its experimental value with the literature value. All QCM-D experiments were conducted at $37{ }^{\circ} \mathrm{C}$, and stable baselines were achieved prior to the addition of inhibitors. For each inhibitor, we carefully selected six to seven different concentrations, which cover the range that allows the attainment of a reasonable dose-response curve and a reliable $\mathrm{IC}_{50}$ value. Although variations often occur for cell-based assays, we made an effort to minimize the impact of any potential variations on the outcomes of the study by conducting QCM-D experiments with cells from at least three separate cultures and fluorescence imaging experiments with cells from two separate cultures. In addition, carefully designed control experiments were performed under individual conditions. For the dose response studies, the variations did not significantly alter the determination of $\mathrm{IC}_{50}$ values.

\subsection{Inhibition of the EGF-Induced Time-Dependent Cellular Responses: QCM-D Measurements}

Figure 1(A) shows the results of a typical dose-response inhibition of the EGF-induced cellular response measured with the QCM-D. In this experiment, seven different doses (0, 10, 20, 50, 100, 200, and $400 \mathrm{nM}$ ) of PD158780, a potent inhibitor of EGFR tyrosine kinase [51] were introduced into confluent monolayers of mutant MCF-10A cells on QCM-D sensor crystals. After incubation at $37{ }^{\circ} \mathrm{C}$ for $40 \mathrm{~min}$, each assay solution was replaced with a solution of $10 \mathrm{nM}$ EGF. The $\Delta D$-responses at the order of overtone, $n=3$, were recorded at $37^{\circ} \mathrm{C}$ for $3 \mathrm{~h}$. Although the QCM-D is able to provide simultaneous measurements of both $\Delta D$ - and $\Delta f$-responses, we focused on the $\Delta D$-responses because it is a far more sensitive measure of the EGF-induced cellular response than the $\Delta f$-response [39].

In Figure 1(A), the $\Delta D$-response curve labeled $0 \mathrm{nM}$ PD158780 represents the time-dependent profile of mutant MCF10A cells responding to $10 \mathrm{nM}$ EGF without the presence of the inhibitor. Immediately upon the addition of EGF, this $\Delta D$-response curve exhibits a sharp upward spike, which 
was an artifact of the mechanical perturbation caused by adding a liquid to the cell layer. After the initial spike, the $\Delta D$-response curve shows a rapid decline that continues until $\sim 25$ min, which corresponds to a rapid cell de-adhesion. At $\sim 40 \mathrm{~min}$, the rate of this decline begins to decrease until the valley of the $\Delta D$-response is reached at $\sim 65 \mathrm{~min}$. The response curve then maintains a virtually constant response from the valley for the next 15 to $20 \mathrm{~min}$. The period from 40 to $80 \mathrm{~min}$ is considered to be the transition phase of the $\Delta D$-response. After the transition, the $\Delta D$-response begins a slow but steady increase for the next $80 \mathrm{~min}$, which corresponds to cell re-adhesion. The response profile of mutant MCF10A cells typically shows a more rapid de-adhesion and slower re-adhesion compared with that of the wildtype cells [41].

Figure 1. Real-time quartz crystal microbalance with dissipation monitoring (QCM-D) measurements (at the order of overtone $n=3$ ) of the $\Delta D$-responses of mutant MCF-10A cells to $10 \mathrm{nM}$ epidermal growth factor (EGF) at $37{ }^{\circ} \mathrm{C}$. The corresponding sequential EGF-induced de-adhesion processes were indicated. (A) The $\Delta D$-responses of the cells were suppressed by PD158780, a known inhibitor of EGFR tyrosine kinase, at various doses $(0,10,20,50,100,200$, and $400 \mathrm{nM})$. (B) The $\Delta D$-responses of the cells were suppressed by cytochalasin $\mathrm{D}$, a known inhibitor of actin polymerization, at various doses $(0,0.1,0.3,0.6,1,2$, and $4 \mu \mathrm{M})$. (C) The $\Delta D$-responses of the cells were suppressed by L779450, a known inhibitor of Raf kinase in the mitogen-activated protein kinase/extracellular signal-regulated kinase (MAPK/ERK) pathway, at various doses $(0,0.1,0.5,5,8$, and $10 \mu \mathrm{M})$. (D) The $\Delta D$-responses of the cells were suppressed by LY294002, a known inhibitor of PI3K in the PI3K pathway, at various doses $(0,0.5,1,3$, $5,8$, and $10 \mu \mathrm{M})$. (E) The $\Delta D$-responses of the cells were increased by U73122, a known inhibitor of phospholipase C (PLC) $\gamma$ in the PLC pathway, at various doses $(0,0.5,1,5,8$, and $10 \mu \mathrm{M}$ ).
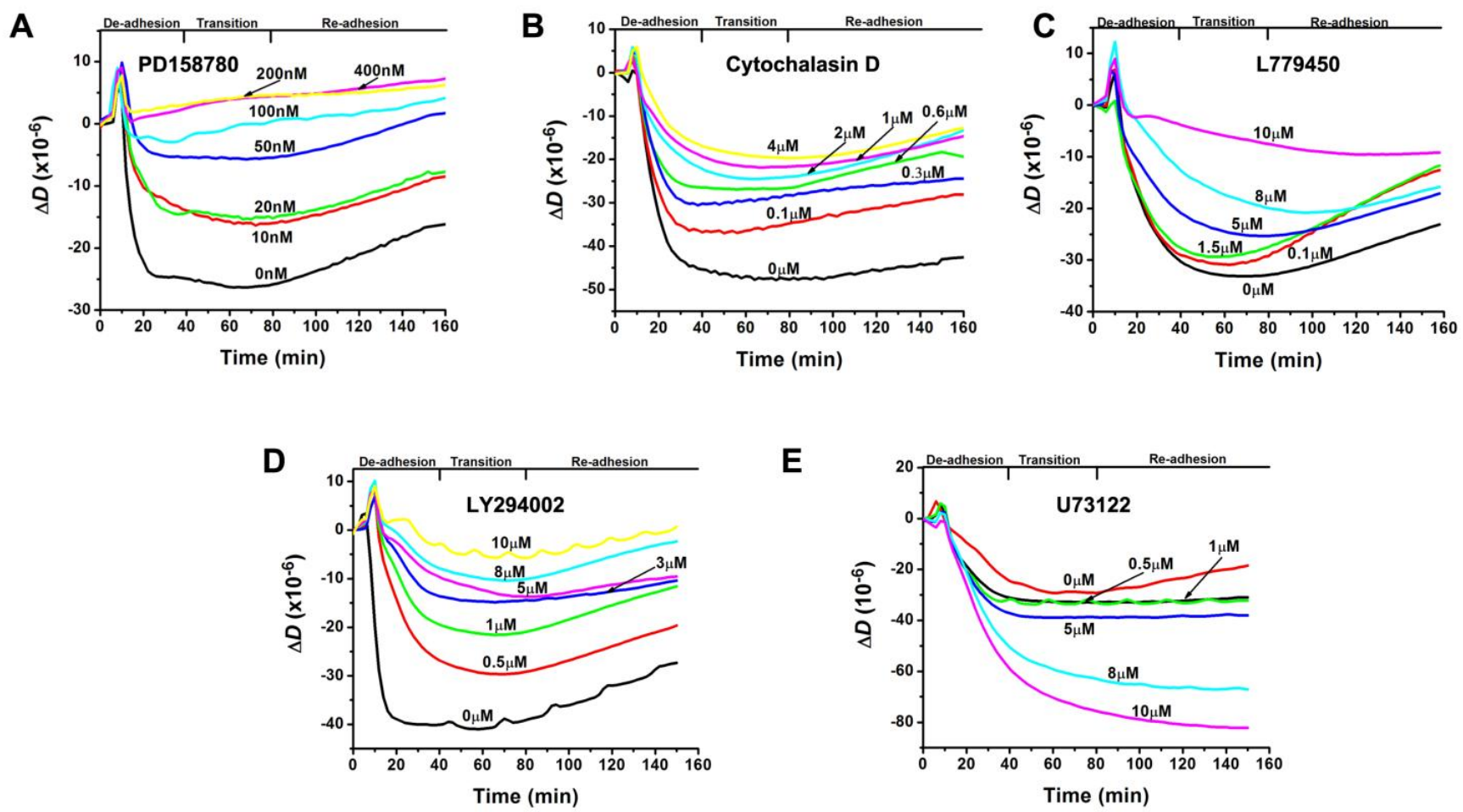

E

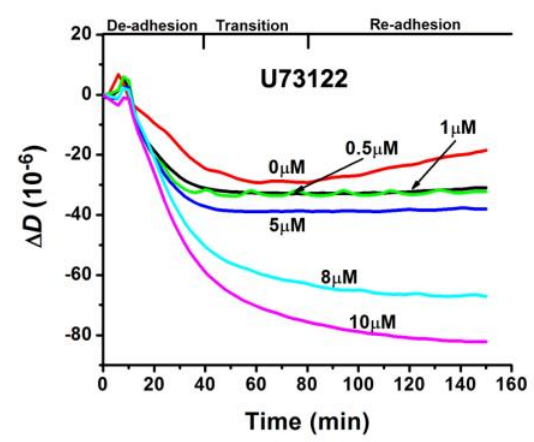


In the presence of PD158780, the amplitude of each EGF-induced $\Delta D$-response curve was substantially reduced, and the higher the concentrations of the inhibitor, the greater the reduction of the amplitudes of the EGF-induced $\Delta D$-response. At the two highest concentrations (200 and $400 \mathrm{nM}$ ), the EGF-induced $\Delta D$-responses were completely abolished, as evidenced by their nearly zero amplitudes (Figure 1(A)). All of these observed changes to the EGF-induced $\Delta D$-responses indicate an essential role of the EGFR tyrosine kinase in regulation of cell de-adhesion.

Figure 1(B) shows the inhibitory effect of cytochalasin $\mathrm{D}$ on the EGF-induced $\Delta D$-response. Cytochalasin D (CD), is a potent, cell-permeable inhibitor of actin polymerization and is capable of attenuating the remodeling of the actin filament [52]. When the cells were pretreated with $\mathrm{CD}$, the EGF-induced $\Delta D$-response was substantially suppressed (Figure 1(B)), confirming that cytoskeleton remodeling is associated with the observed $\Delta D$-response to EGF. This further underscores the connection between cytoskeleton remodeling and cell signaling and trafficking in general $[53,54]$.

Figure $1(\mathrm{C}-\mathrm{E})$ show the results of the inhibition of downstream signaling pathways of EGFR including the MAPK/ERK, PI3K, and PLC pathways. These pathways, which are responsible for regulating the assembly and disassembly of focal adhesions that lead to changes in cell adhesion [55-57], have been the targets for therapeutic development in recent years [58-60]. To probe these pathways, we pretreated cells with L779450 [61], a potent cell-permeable inhibitor of Raf kinase in the MAPK/ERK pathway, with LY294002, a potent inhibitor of PI3K in the PI3K pathway [62], or with U73122, a potent inhibitor of PLC $\gamma$ (an isotype of phospholipase) in the PLC pathway [63]. It is evident that the EGF-induced $\Delta D$-responses were altered by the inhibitors in a dose-dependent manner (Figure 1(C-E)). For both L779450 (Figure 1(C)) and LY294002 (Figure 1(D)), the amplitudes of the EGF-induced $\Delta D$-responses were reduced, suggesting that the MAPK/ERK and PI3K pathways are responsible for promoting the EGF-induced cellular response in mutant MCF-10A cells. These results are in line with the previous reports that the MAPK/ERK pathway is responsible for the EGF-induced disassembly of focal adhesion in fibroblasts [5] and the PI3K pathway may alter cell adhesion through its downstream effectors including small GTPase Rho A, and/or through crosstalk with the MAPK/ERK pathway [55]. For U73122, the amplitudes of the EGF-induced $\Delta D$-responses were not reduced. Instead, they increased as the concentration of U73122 increased (Figure 1(E)), suggesting an enhanced cell de-adhesion upon the inhibition of the PLC pathway, a pathway known to play an important role in EGF-mediated cell adhesion and motility [64].

Interestingly, the inhibition of each pathway led to distinct features of the time-dependent dose-response profiles, shown in Figure $1(\mathrm{C}-\mathrm{E})$. For example, when the MAPK/ERK pathway was inhibited by L779450, the portions of the EGF-induced response curves corresponding to the initial rapid de-adhesion (first $20 \mathrm{~min}$ ) remained close to each other for most inhibitor concentrations (Figure 1(C)). By contrast, all inhibitory response curves were well separated from each other during the same period of time (first $20 \mathrm{~min}$ ) when the PI3K pathway was inhibited by LY294002 (Figure 1(D)). While the re-adhesion still occurred when either the MAPK/ERK or PI3K pathway was inhibited (Figure 1(C,D)), re-adhesion was clearly absent when the PLC pathway was inhibited (Figure 1(E)). These distinct features of the inhibition profiles can be regarded as the "signatures" of the cellular response of individual pathways, i.e., a unique form of the pathway-dependent phenotypic response [65-67]. These signatures could potentially be very informative by providing mechanistic insights into the regulation of the EGF-induced cell de-adhesion in mutant MCF10A cells. 


\subsection{Inhibition of the EGF-Induced Time-Dependent Cellular Responses: Fluorescence Imaging}

Label-free biosensors are capable of producing real-time, measurable signals by capturing time-dependent cellular responses, which often result from more than one of many possible changes that relate to cellular morphology, adhesion, ion distribution, and mass distribution $[16,17,67]$. Because these signals are the results of the collected effects within cells, individual contributing molecular components and their associated cellular processes are often difficult to identify. Thus, the label-free biosensor signals are commonly referred to as "black box" assay readouts [68].

In the previous work, we had examined the time-dependent cell de-adhesion in response to EGF with fluorescence imaging. We were able to establish a quantitative relationship between the $\Delta D$-response obtained from the QCM-D and the level of focal adhesions, quantified with measurements of the areal density of immunostained vinculin [39]. To our knowledge, this is one of the few studies done to make a quantitative connection between a cell-based biosensor signal and a specific cellular component and its associated cellular process. Following the same strategy in the present study, we intended to verify the inhibitory effect of each inhibitor on mutant MCF10A cells and further establish the connection between the time-dependent $\Delta D$-response and the dynamic restructuring of focal adhesions.

Figure 2. Fluorescence images of immunostained vinculin within focal adhesions of mutant MCF10A cells in response to $10 \mathrm{nM}$ EGF. Examples of focal adhesions are indicated with arrows. Scale bar: $10 \mu \mathrm{m}$. The cells had been pretreated with an inhibitor described in (B-G). (A) Quantitation of the areal densities of stained vinculin in relative fluorescence units (RFU) as a measure for the level of focal adhesions (mean \pm SEM; $\mathrm{n}=10)$ in $(\mathrm{B}-\mathrm{G})$. (B) The control without the presence of an inhibitor. (C) $100 \mathrm{nM}$ PD158780. (D) $1 \mu \mathrm{M}$ cytochalasin D. (E) $10 \mu \mathrm{M}$ L77945. (F) $10 \mu \mathrm{M}$ LY294002. (G) $5 \mu \mathrm{M}$ U73122. The difference in fluorescence intensities between the control and each inhibited sample is significant as indicated by $\mathrm{P}<0.001$ for all five inhibited samples.

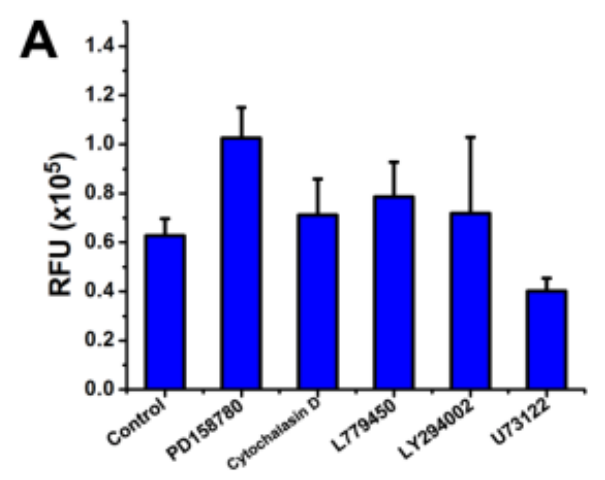

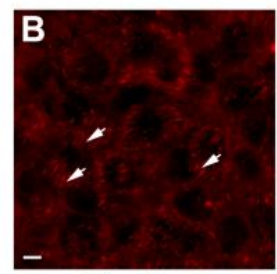

Control

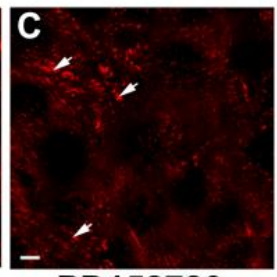

PD158780
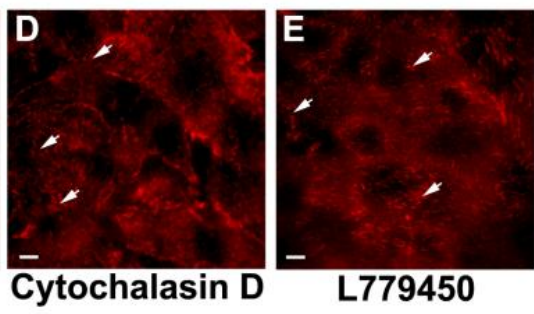

L779450

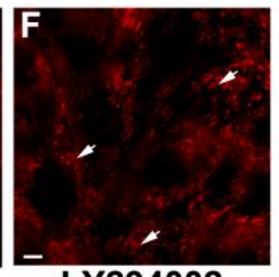

LY294002

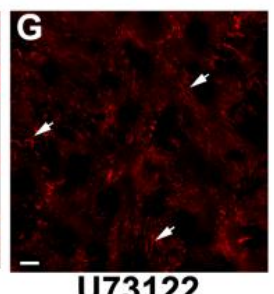

U73122 
First we examined the inhibitory effect of each inhibitor on the EGF-induced changes in level of focal adhesions. Figure $2(B-G)$ show fluorescence images of immunostained vinculin within focal adhesions of mutant MCF10A cells. Each image was taken from the cell sample that had been pretreated with a specific inhibitor (or the assay buffer for the control) for $40 \mathrm{~min}$ and then exposed to $10 \mathrm{nM}$ EGF for $60 \mathrm{~min}$. The overall comparison based on the quantitation of fluorescence intensities of stained vinculin is shown in Figure 2(A). With the presence of the inhibitors including PD158780 (100 nM, Figure 2(C)), cytochalasin D (1 $\mu \mathrm{M}$, Figure 2(D)), L779450 (10 $\mu \mathrm{M}$, Figure 2(E)), and LY294002 (10 $\mu \mathrm{M}$, Figure 2(F)), the cells exhibit slightly higher fluorescence intensities of vinculin compared to the control which does not have any inhibitors (Figure 2(B)). These results indicate that these inhibitors enhanced the cell adhesion by suppressing the EGF-induced de-adhesion, which is consistent with the observed reduction of the amplitudes of the $\Delta D$-responses in Figure $1(\mathrm{~A}-\mathrm{D})$. For the sample that had been pretreated with $5 \mu \mathrm{M}$ U73122 (Figure $2(\mathrm{G})$ ), the cells exhibit a slightly lower fluorescence intensity compared to the control (Figure 2(A)), which is consistent with the increasing amplitudes of the $\Delta D$-responses in the presence of U73122 (Figure 1(E)), a sign of an enhanced cell de-adhesion.

Next we investigated the time-dependent effect of each inhibitor by focusing on changes in areal density of focal adhesions (Figure 3). Figure 3(AC-AG) show fluorescence images of immunostained vinculin within focal adhesions of mutant MCF10A cells that had been pretreated with $100 \mathrm{nM}$ PD158780 and then exposed to $10 \mathrm{nM}$ EGF for various times (0, 30, 60, 100, and $150 \mathrm{~min})$. Because of the high fluorescence background, some of the images in Figure 3 look brighter than others, even though the intensities of immunostained vinculin in those images may not be necessarily higher than others. The appearance of dark holes enclosed within bright boundaries was probably due to immunostained vinculin at sites of cell-cell contact. Prior to exposure to EGF ( 0 min), numerous prominent focal adhesions are present as short bright streaks of vinculin in both the central regions and the peripheries of the cells that had been pretreated with $100 \mathrm{nM}$ PD158780 (Figure 3(AC)). Upon exposure to $10 \mathrm{nM}$ EGF for $30 \mathrm{~min}$, the cells exhibit fewer, smaller, and less intense spots of stained vinculin (Figure 3(AD)), indicating a lowered level of focal adhesions. A 60-min exposure to EGF further diminished the spots of stained vinculin in size and number, as shown in Figure 3(AE). However, a longer exposure to EGF, e.g., $100 \mathrm{~min}$, did not cause any further diminution of stained vinculin compared with the 60 min exposure. In fact, they show a slight increase in both size and number, which is indicative of an increase in level of focal adhesions (Figure 3(AF)). After a $150 \mathrm{~min}$ exposure to EGF, stained vinculin spots (Figure 3(AG)) become even more noticeable compared with the 100-min exposure. Overall, when exposed to EGF, a monolayer of mutant MCF10A cells underwent time-dependent restructuring of focal adhesions, which corresponds to de-adhesion, transition (around $60 \mathrm{~min}$ ), and re-adhesion. This pattern is consistent with the one revealed with the QCM-D measurements in Figure 1(A). The cells in the presence of other inhibitors in this study also showed similar patterns to that of PD158780 (Figure 3).

For each inhibitor, the levels of focal adhesions are quantified and summarized as a function of time in bar graph form in Figure 3(AA,BA,CA,DA,EA), respectively. The time-dependent changes in level of focal adhesions were fit with the corresponding $\Delta D$-response curve. Reasonably good fits are obtained for all five inhibitors, confirming the correlation between the magnitude of the $\Delta D$-response 
and the level of focal adhesions, and suggesting that the change in cell adhesion, more specifically, the restructuring of focal adhesions can be assessed based on the time-dependent change in $\Delta D$-response.

Figure 3. Time dependent relationship between changes in energy dissipation factor and vinculin immunofluorescence staining within focal adhesions of mutant MCF10A cells in response to $10 \mathrm{nM}$ EGF. Examples of focal adhesions are indicated with arrows. Scale bar: $10 \mu \mathrm{m}$. The cells had been pretreated with the following inhibitors: $100 \mathrm{nM}$ PD158780 (row A), $1 \mu \mathrm{M}$ cytochalasin D (row B), $10 \mu \mathrm{M}$ L779450 (row C), $10 \mu \mathrm{M}$ LY294002 (row D), and $5 \mu \mathrm{M}$ U73122 (row E). (AA), (BA), (CA), (DA), and (EA) Quantitation of the areal densities of stained vinculin in relative fluorescence units (RFU) as a measure of focal adhesions (mean $\pm \mathrm{SEM} ; \mathrm{n}=10$ ). A strong correlation is shown between the normalized $\Delta D$-response and the normalized RFU of focal adhesions. To ensure that the values of RFU and $\Delta D$ could be compared, each of the values was normalized, i.e., was divided by the range covered. For each quantity, the range was taken as the highest value (at $0 \mathrm{~min}$ ) minus the lowest value (at $60 \mathrm{~min}$ ). All correlations are highly statistically significant ( $\mathrm{p}<0.005$ ). In each of rows (A) to (E), column (B) shows the fluorescence images of focal adhesions in a monolayer of cells prior to inhibition, labeled as uninhibited. Columns (C) to $(\mathrm{G})$ show the fluorescence images of focal adhesions in a monolayer of cells after being exposed first to the inhibitor for $40 \mathrm{~min}$, then to $10 \mathrm{nM}$ EGF for: (C) $0 \mathrm{~min}$, (D) $30 \mathrm{~min}$, (E) $60 \mathrm{~min}$, and (F) $100 \mathrm{~min}$, and (G) $150 \mathrm{~min}$.

\section{PD158780}

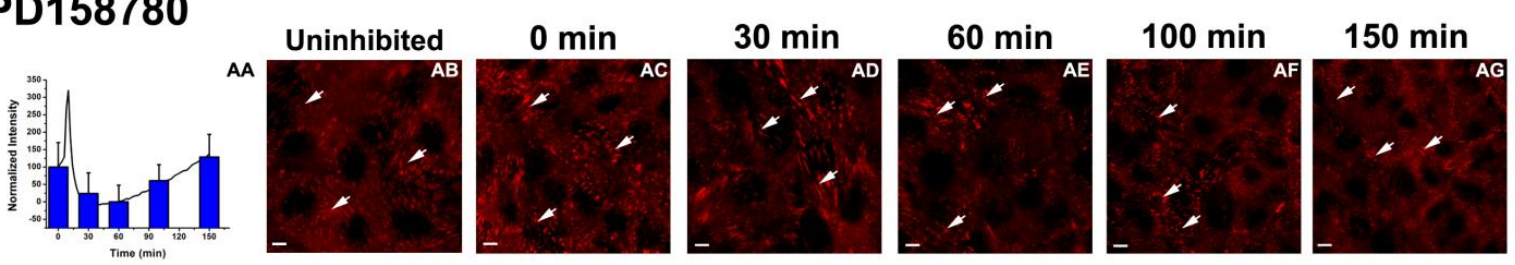

\section{Cytochalasin D}
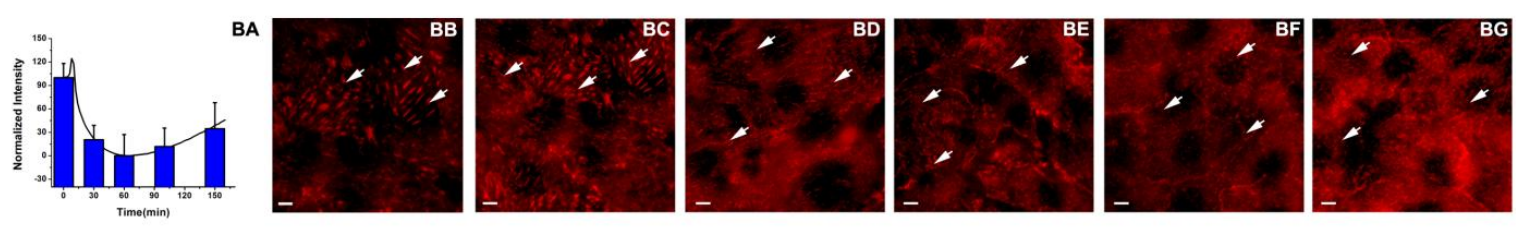

\section{L779450}
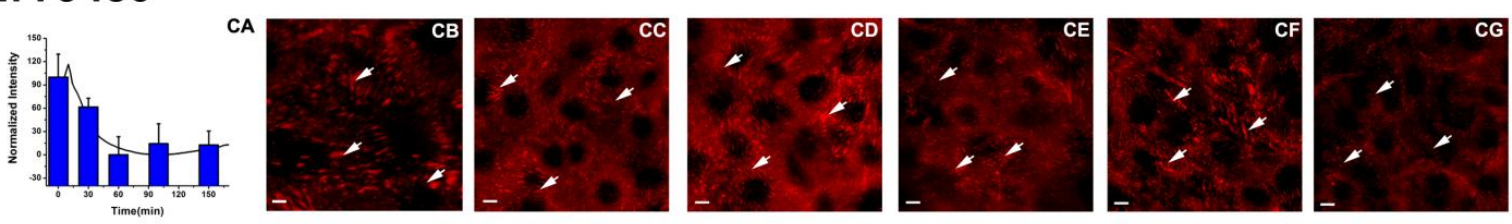

\section{LY294002}
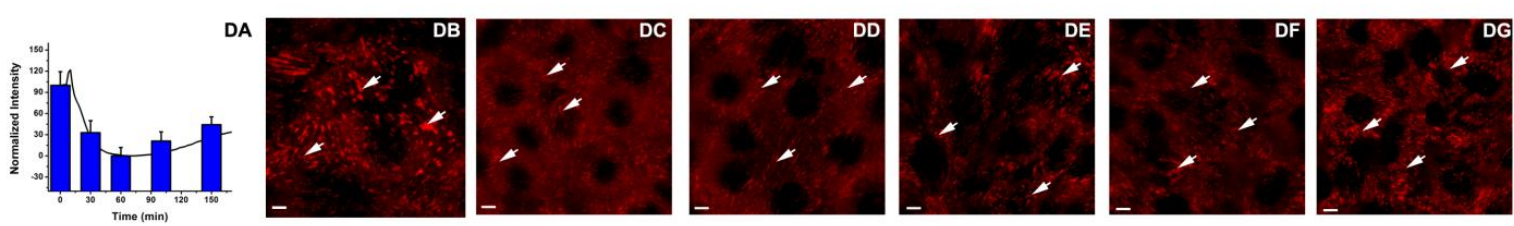
Figure 3. Cont.

\section{U73122}
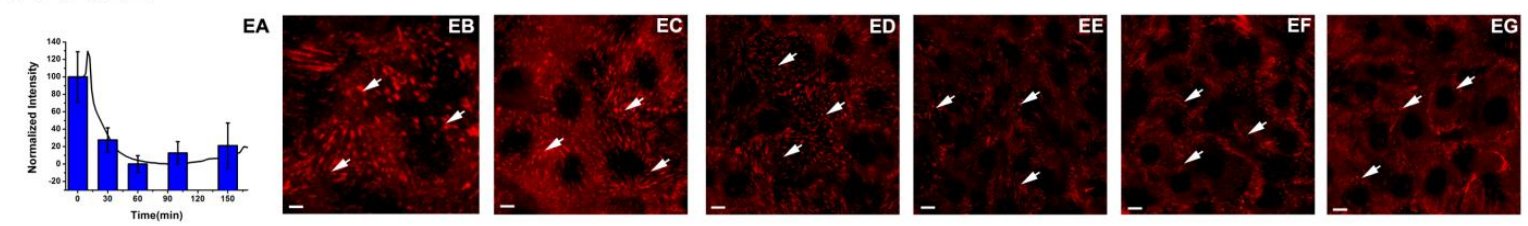

\subsection{Inhibition of the EGF-Induced Time-Dependent Cellular Responses: Potencies of the Inhibitors}

By far we have shown that the QCM-D has the capability of measuring the inhibitory effects of small molecule inhibitors on the EGF-induced cellular response. To have a more quantitative assessment of the effectiveness of the QCM-D, we determined the $\mathrm{IC}_{50}$ value for each of the five inhibitors. By comparing the experimental value with the reported value, we could assess the sensitivity and particularly the reliability of the QCM-D as a screening tool.

Figure 4. The amplitudes of EGF-induced $\Delta D$-responses at $40 \mathrm{~min}$ as a function of inhibitor concentrations. The amplitude is defined as the absolute value of the difference between the experimental value and the control value. The data, derived from the average of at least three sets of independent data, was fit with the dose-response function. The resulting $\mathrm{IC}_{50}$ values are also listed in Table 1. (A) PD158780. $\mathrm{IC}_{50}=64 \pm 30 \mathrm{nM}$.

(B) cytochalasin D. $\mathrm{IC}_{50}=0.18 \pm 0.11 \mu \mathrm{M}$. (C) L779450. $\mathrm{IC}_{50}=1.0 \pm 0.6 \mu \mathrm{M}$.

(D) $\mathrm{LY} 294002 . \mathrm{IC}_{50}=1.1 \pm 0.5 \mu \mathrm{M}$. (E) U73122. $\mathrm{IC}_{50}=2.5 \pm 0.9 \mu \mathrm{M}$.
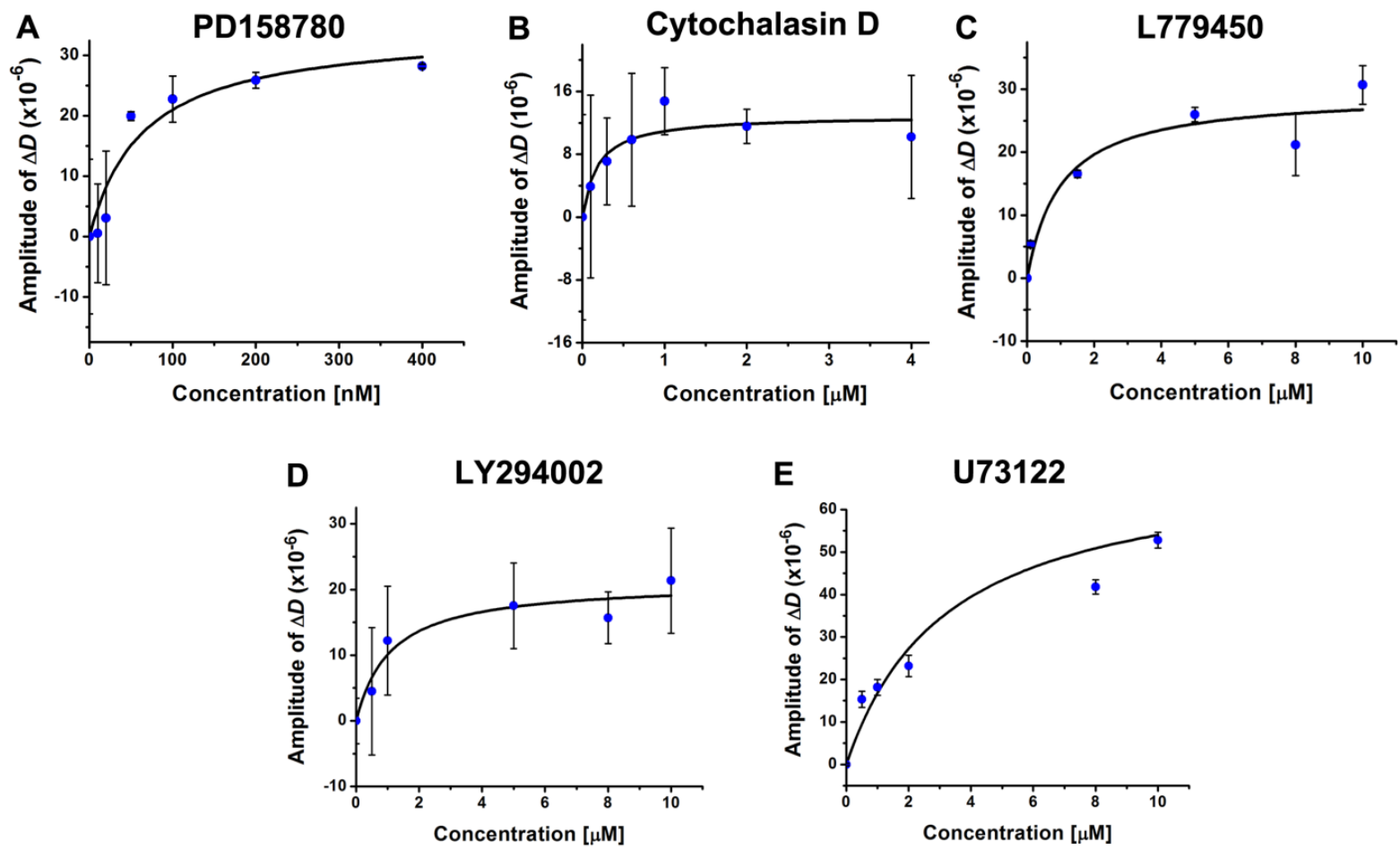

The $\mathrm{IC}_{50}$ value for each inhibitor was derived based on its dose dependence determined by fitting the amplitudes of the $\Delta D$-response values at $40 \mathrm{~min}$ as a function of the inhibitor concentrations (Figure 4). These values obtained from the QCM-D measurements along with the values reported in 
the literature are listed in Table 1 . The reported $\mathrm{IC}_{50}$ value for cytochalasin $\mathrm{D}$ was determined based on its inhibitory effect on the mechanical properties of the cell sample [69]. All other values were determined based on the in vivo inhibitory effects on the target enzymes in cell samples [62,70-72]. It is apparent that the experimental values are in strong agreement with the reported values (Table 1), which strongly supports the notion that the QCM-D has the sensitivity and reliability to be potentially utilized as a sensing platform for drug screening. To achieve this goal, increasing the throughput capacity of the QCM-D would be the next critical step. In addition, establishing the technical advantage of the QCM-D compared to other sensor technologies would also be important.

Table 1. Comparison of $\mathrm{IC}_{50}$ values of the inhibitors.

\begin{tabular}{lll}
\hline Inhibitor & IC $_{50}$ (QCM-D) & IC $_{50}$ (literature) \\
\hline PD158780 & $64 \pm 30 \mathrm{nM}$ & $52 \mathrm{nM} \mathrm{[70]}$ \\
cytochalasin D & $0.18 \pm 0.11 \mu \mathrm{M}$ & $0.25 \mu \mathrm{M}[69]$ \\
L779450 & $1.0 \pm 0.6 \mu \mathrm{M}$ & $1 \mu \mathrm{M}[71]$ \\
$\mathrm{LY} 294002$ & $1.1 \pm 0.5 \mu \mathrm{M}$ & $1.4 \mu \mathrm{M}[62]$ \\
$\mathrm{U} 73122$ & $2.5 \pm 0.9 \mu \mathrm{M}$ & $1-2.1 \mu \mathrm{M}[72]$ \\
\hline
\end{tabular}

\section{Conclusions}

In this report, we have employed a label-free acoustic sensor, the QCM-D, to track the EGF-induced response of mutant MCF10A cells under various inhibitory conditions. We have identified a complex cell de-adhesion process, which can be distinctly altered by inhibitors of signaling pathways and cytoskeleton formation in a dose-dependent manner. The dose dependencies of the inhibitors provide $\mathrm{IC}_{50}$ values which are in strong agreement with the values reported in the literature, demonstrating the sensitivity and reliability of the QCM-D as a screening tool. Using immunofluorescence imaging, we have also verified the quantitative relationship between the $\Delta D$-response and the level of focal adhesions under those inhibitory conditions. Such a correlation suggests that the dynamic restructuring of focal adhesions can be assessed based on the time-dependent change in $\Delta D$-response. Overall, this report has shown that the QCM-D has the potential to become an effective sensing platform for screening therapeutic agents that target signaling and cytoskeletal proteins.

\section{Acknowledgments}

We thank Mauricio Reginato for providing us mutant MCF10A cells that overexpress EGFR.

\section{References}

1. Carpenter, G. Receptors for epidermal growth factor and other polypeptide mitogens. Annu. Rev. Biochem. 1987, 56, 881-914.

2. Lemmon, M.A.; Schlessinger, J. Cell signaling by receptor tyrosine kinases. Cell. 2010, 141, $1117-1134$.

3. Scaltriti, M.; Baselga, J. The epidermal growth factor receptor pathway: A model for targeted therapy. Clin. Cancer Res. 2006, 12, 5268-5272. 
4. Osaki, M.; Oshimura, M.; Ito, H. PI3K-Akt pathway: Its functions and alterations in human cancer. Apoptosis 2004, 9, 667-676.

5. Xie, H.; Pallero, M.A.; Gupta, K.; Chang, P.; Ware, M.F.; Witke, W.; Kwiatkowski, D.J.; Lauffenburger, D.A.; Murphy-Ullrich, J.E.; Wells, A. EGF receptor regulation of cell motility: EGF induces disassembly of focal adhesions independently of the motility-associated PLCgamma signaling pathway. J. Cell. Sci. 1998, 111, 615-624.

6. Zandi, R.; Larsen, A.B.; Andersen, P.; Stockhausen, M.-T.; Poulsen, H.S. Mechanisms for oncogenic activation of the epidermal growth factor receptor. Cell. Signal. 2007, 19, 2013-2023.

7. Sebastian, S.; Settleman, J.; Reshkin, S.J.; Azzariti, A.; Bellizzi, A.; Paradiso, A. The complexity of targeting EGFR signalling in cancer: From expression to turnover. BBA-Rev. Cancer 2006, $1766,120-139$.

8. Normanno, N.; De Luca, A.; Bianco, C.; Strizzi, L.; Mancino, M.; Maiello, M.R.; Carotenuto, A.; De Feo, G.; Caponigro, F.; Salomon, D.S. Epidermal growth factor receptor (EGFR) signaling in cancer. Gene 2006, 366, 2-16.

9. Oda, K.; Matsuoka, Y.; Funahashi, A.; Kitano, H. A comprehensive pathway map of epidermal growth factor receptor signaling. Mol. Syst. Biol. 2005, doi: 10.1038/msb4100014.

10. Jordan, J.D.; Landau, E.M.; Iyengar, R. Signaling networks: The origins of cellular multitasking. Cell 2000, 103, 193-200.

11. Kholodenko, B.N. Cell-signalling dynamics in time and space. Nat. Rev. Mol. Cell Biol. 2006, 7, $165-176$.

12. Denholm, E.M.; Gerald, P.S. Differential effects of two fluorescent probes on macrophage migration as assessed by manual and automated methods. Cytometry 1995, 19, 366-369.

13. Abbitt, K.B.; Rainger, G.E.; Nash, G.B. Effects of fluorescent dyes on selectin and integrin-mediated stages of adhesion and migration of flowing leukocytes. J. Immunol. Meth. 2000, 239, 109-119.

14. Xi, B.; Naichen, Y.; Xiaobo, W.; Xiao, X.; Yama, A. The application of cell-based label-free technology in drug discovery. Biotechnol. J. 2008, 3, 484-495.

15. Fang, Y. Label-free biosensors for cell biology. Int. J. Electrochem. 2011, doi: 10.4061/2011/460850.

16. Cooper, M.A. Non-optical screening platforms: The next wave in label-free screening? Drug Discov. Today 2006, 11, 1068-1074.

17. Cooper, M.A. Optical biosensors: Where next and how soon? Drug Discov. Today 2006, 11, 1061-1067.

18. Fang, Y. Non-invasive optical biosensor for probing cell signaling. Sensors 2007, 7, 2316-2329.

19. Fang, Y.; Ferrie, A.M.; Fontaine, N.H.; Mauro, J.; Balakrishnan, J. Resonant waveguide grating biosensor for living cell sensing. Biophys. J. 2006, 91, 1925-1940.

20. Pattnaik, P. Surface plasmon resonance. Appl. Biochem. Biotechnol. 2005, 126, 79-92.

21. McDonnell, J.M. Surface plasmon resonance: Towards an understanding of the mechanisms of biological molecular recognition. Curr. Opin. Chem. Biol. 2001, 5, 572-577.

22. Giaever, I.; Keese, C.R. A morphological biosensor for mammalian cells. Nature 1993, 366, 591-592.

23. Chen, J.Y.; Li, M.; Penn, L.S.; Xi, J. Real-time and label-free detection of cellular response to signaling mediated by distinct subclasses of epidermal growth factor receptors. Anal. Chem. 2011, $83,3141-3146$. 
24. Atienza, J.M.; Yu, N.; Wang, X.; Xu, X.; Abassi, Y. Label-free and real-time cell-based kinase assay for screening selective and potent receptor tyrosine kinase inhibitors using microelectronic sensor array. J. Biomol. Screen. 2006, 11, 634-643.

25. Fang, Y.; Ferrie, A.M.; Fontaine, N.H.; Yuen, P.K. Characteristics of dynamic mass redistribution of epidermal growth factor receptor signaling in living cells measured with label-free optical biosensors. Anal. Chem. 2005, 77, 5720-5725.

26. Liu, F.; Zhang, J.; Deng, Y.; Wang, D.; Lu, Y.; Yu, X. Detection of EGFR on living human gastric cancer BGC823 cells using surface plasmon resonance phase sensing. Sens. Actuator. B: Chem. 2011, 153, 398-403.

27. Fredriksson, C.; Kihlman, S.; Rodahl, M.; Kasemo, B. The piezoelectric quartz crystal mass and dissipation sensor: A means of studying cell adhesion. Langmuir 1998, 14, 248-251.

28. Rodahl, M.; Hook, F.; Fredriksson, C.; Keller, C.A.; Krozer, A.; Brzezinski, P.; Voinova, M.; Kasemo, B. Simultaneous frequency and dissipation factor QCM measurements of biomolecular adsorption and cell adhesion. Faraday Discuss. 1997, 107, 229-246.

29. Dixon, M.C. Quartz crystal microbalance with dissipation monitoring: Enabling real-time characterization of biological materials and their interactions. J. Biomol. Tech. 2008, 19, 151-158.

30. Marx, K.A. The quartz crystal microbalance and the electrochemical QCM: Applications to studies of thin polymer films, electron transfer systems, biological macromolecules, biosensors, and cells. Piezoelectric Sens. 2007, 5, 371-424.

31. Matsuda, T.; Kishida, A.; Ebato, H.; Okahata, Y. Novel instrumentation monitoring in situ platelet adhesivity with a quartz crystal microbalance. ASAIO J. 1992, 38, M171-M173.

32. Redepenning, J.; Schlesinger, T.K.; Mechalke, E.J.; Puleo, D.A.; Bizios, R. Osteoblast attachment monitored with a quartz crystal microbalance. Anal. Chem. 1993, 65, 3378-3381.

33. Gryte, D.M.; Michael, D.W.; Wei-Shou, H. Real-time measurement of anchorage-dependent cell adhesion using a quartz crystal microbalance. Biotechnol. Progr. 1993, 9, 105-108.

34. Janshoff, A.; Wegener, J.; Sieber, M.; Galla, H.J. Double-mode impedance analysis of epithelial cell monolayers cultured on shear wave resonators. Eur. Biophys. J. 1996, 25, 93-103.

35. Wegener, J.; Janshoff, A.; Galla, H.J. Cell adhesion monitoring using a quartz crystal microbalance: Comparative analysis of different mammalian cell lines. Eur. Biophys. J. 1998, 28, 26-37.

36. Heitmann, V.; Reiss, B.; Wegener, J. The quartz crystal microbalance in cell biology: Basics and applications. Piezoelectric Sens. 2007, 5, 303-338.

37. Nimeri, G.; Fredriksson, C.; Elwing, H.; Liu, L.; Rodahl, M.; Kasemo, B. Neutrophil interaction with protein-coated surfaces studied by an extended quartz crystal microbalance technique. Colloid. Surface. B 1998, 11, 255-264.

38. Saitakis, M.; Gizeli, E. Acoustic sensors as a biophysical tool for probing cell attachment and cell/surface interactions. Cell. Mol. Life Sci. 2012, 69, 357-371.

39. Chen, J.Y.; Shahid, A.; Garcia, M.P.; Penn, L.S.; Xi, J. Dissipation monitoring for assessing EGF-induced changes of cell adhesion. Biosens. Bioelectron. 2012, 38, 375-381.

40. Yang, R.; Chen, J.Y.; Xi, N.; Lai, K.W.C.; Qu, C.; Fung, C.K.M.; enn, L.S.; Xi, J. Characterization of mechanical behavior of an epithelial monolayer in response to epidermal growth factor stimulation. Exp. Cell Res. 2012, 318, 521-526. 
41. Garcia, M.P.; Shahid, A.; Chen, J.Y.; Xi, J. Effects of the expression level of epidermal growth factor receptor on the ligand-induced restructuring of focal adhesions: A QCM-D study. Anal Bioanal Chem. 2012, in press.

42. Reginato, M.J.; Mills, K.R.; Paulus, J.K.; Lynch, D.K.; Sgroi, D.C.; Debnath, J.; Muthuswamy, S.K.; Brugge, J.S. Integrins and EGFR coordinately regulate the pro-apoptotic protein Bim to prevent anoikis. Nat. Cell Biol. 2003, 5, 733-740.

43. Dei Tos, A.P.; Ellis, I. Assessing epidermal growth factor receptor expression in tumours: What is the value of current test methods. Eur. J. Cancer 2005, 41, 1383-1392.

44. Hynes, R.O. Integrins: Bidirectional, allosteric signaling machines. Cell 2002, 110, 673-687.

45. Wheelock, M.J.; Johnson, K.R. Cadherins as modulators of cellular phenotype. Annu. Rev. Cell Dev. Biol. 2003, 19, 207-235.

46. Arteaga, C.L. Epidermal growth factor receptor dependence in human tumors: More than just expression. Oncologist 2002, 7, 31-39.

47. Balaban, N.Q.; Schwarz, U.S.; Riveline, D.; Goichberg, P.; Tzur, G.; Sabanay, I.; Mahalu, D.; Safran, S.; Bershadsky, A.; Addadi, L.; Geiger, B. Force and focal adhesion assembly: A close relationship studied using elastic micropatterned substrates. Nat. Cell Biol. 2001, 3, 466-472.

48. Beningo, K.A.; Dembo, M.; Kaverina, I.; Small, J.V.; Wang, Y.-1. Nascent focal adhesions are responsible for the generation of strong propulsive forces in migrating fibroblasts. J. Cell Biol. 2001, 153, 881-888.

49. Gallant, N.D.; Michael, K.E.; García, A.J. Cell adhesion strengthening: contributions of adhesive area, integrin binding, and focal adhesion assembly. Mol. Biol. Cell 2005, 16, 4329-4340.

50. Tan, J.L.; Tien, J.; Pirone, D.M.; Gray, D.S.; Bhadriraju, K.; Chen, C.S. Cells lying on a bed of microneedles: An approach to isolate mechanical force. Proc. Natl. Acad. Sci. USA 2003, 100, 1484-1489.

51. Rewcastle, G.W.; Palmer, B.D.; Thompson, A.M.; Bridges, A.J.; Cody, D.R.; Zhou, H.; Fry, D.W.; McMichael, A.; Denny, W.A. Tyrosine kinase inhibitors. 10. isomeric 4-[(3-bromophenyl) amino]pyrido[d]-pyrimidines are potent ATP binding site inhibitors of the tyrosine kinase function of the epidermal growth factor receptor. J. Med. Chem. 1996, 39, 1823-1835.

52. Schliwa, M. Action of cytochalasin D on cytoskeletal networks. J. Cell Biol. 1982, 92, 79-91.

53. Janmey, P.A. The cytoskeleton and cell signaling: Component localization and mechanical coupling. Physiol. Rev. 1998, 78, 763-781.

54. Papakonstanti, E.A.; Stournaras, C. Cell responses regulated by early reorganization of actin cytoskeleton. FEBS Lett. 2008, 582, 2120-2127.

55. Cain, R.J.; Ridley, A.J. Phosphoinositide 3-kinases in cell migration. Biol. Cell. 2009, 101, 13-29.

56. Allen, F.D.; Asnes, C.F.; Chang, P.; Elson, E.L.; Lauffenburger, D.A.; Wells, A. Epidermal growth factor induces acute matrix contraction and subsequent calpain-modulated relaxation. Wound Repair Regen. 2002, 10, 67-76.

57. Kharait, S.; Tran, K.; Yates, C.; Wells, A. Cell motility in prostate tumor invasion and metastasis. In Cell Motility in Cancer Invasion and Metastasis; Wells, A., Ed.; Springer: Dordrecht, The Netherlands, 2006; Volume 8, pp. 301-338.

58. Workman, P.; Clarke, P.A.; Raynaud, F.I.; van Montfort, R.L.M. Drugging the PI3 kinome: From chemical tools to drugs in the clinic. Cancer Res. 2010, 70, 2146-2157. 
59. Thompson, N.; Lyons, J. Recent progress in targeting the Raf/MEK/ERK pathway with inhibitors in cancer drug discovery. Curr. Opin. Pharmacol. 2005, 5, 350-356.

60. Fabian, M.A.; Biggs, W.H.; Treiber, D.K.; Atteridge, C.E.; Azimioara, M.D.; Benedetti, M.G.; Carter, T.A.; Ciceri, P.; Edeen, P.T.; Floyd, M.; et al. A small molecule-kinase interaction map for clinical kinase inhibitors. Nat. Biotech. 2005, 23, 329-336.

61. Gollob, J.A.; Wilhelm, S.; Carter, C.; Kelley, S.L. Role of Raf kinase in cancer: Therapeutic potential of targeting the Raf/MEK/ERK signal transduction pathway. Semin. Oncol. 2006, 33, 392-406.

62. Vlahos, C.J.; Matter, W.F.; Hui, K.Y.; Brown, R.F. A specific inhibitor of phosphatidylinositol 3-kinase, 2-(4-morpholinyl)-8-phenyl-4H-1-benzopyran-4-one (LY294002). J. Biol. Chem. 1994, 269, 5241-5248.

63. Smith, R.J.; Sam, L.M.; Justen, J.M.; Bundy, G.L.; Bala, G.A.; Bleasdale, J.E. Receptor-coupled signal transduction in human polymorphonuclear neutrophils: Effects of a novel inhibitor of phospholipase C-dependent processes on cell responsiveness. J. Pharmacol. Exp. Ther. 1990, 253, 688-697.

64. Wells, A.; Kassis, J.; Solava, J.; Turner, T.; Lauffenburger, D.A. Growth factor-induced cell motility in tumor invasion. Acta Oncol. 2002, 41, 124-130.

65. Schroder, R.; Janssen, N.; Schmidt, J.; Kebig, A.; Merten, N.; Hennen, S.; Muller, A.; Blattermann, S.; Mohr-Andra, M.; Zahn, S.; et al. Deconvolution of complex G protein-coupled receptor signaling in live cells using dynamic mass redistribution measurements. Nat. Biotech. 2010, 28, 943-949.

66. Fang, Y. Label-free cell-based assays with optical biosensors in drug discovery. Assay Drug Dev. Technol. 2006, 4, 583-595.

67. Fang, Y. Label-free receptor assays. Drug Discovery Today 2010, 7, e5-e11.

68. Rocheville, M.; Jerman, J.C. 7TM pharmacology measured by label-free: A holistic approach to cell signalling. Curr. Opin. Pharmacol. 2009, 9, 643-649.

69. Wakatsuki, T.; Schwab, B.; Thompson, N.C.; Elson, E.L. Effects of cytochalasin D and latrunculin B on mechanical properties of cells. J. Cell Sci. 2001, 114, 1025-1036.

70. Fry, D.W.; Nelson, J.M.; Slintak, V.; Keller, P.R.; Rewcastle, G.W.; Denny, W.A.; Zhou, H.; Bridges, A.J. Biochemical and antiproliferative properties of 4-[Ar(alk)ylamino]pyridopyrimidines, a new chemical class of potent and specific epidermal growth factor receptor tyrosine kinase inhibitor. Biochem. Pharmacol. 1997, 54, 877-887.

71. Shelton, J.G.; Moye, P.W.; Steelman, L.S.; Blalock, W.L.; Lee, J.T.; Franklin, R.A.; McMahon, M.; McCubrey, J.A. Differential effects of kinase cascade inhibitors on neoplastic and cytokine-mediated cell proliferation. Leukemia 2003, 17, 1765-1782.

72. Xie, W.; Peng, H.; Zalkow, L.H.; Li, Y.-H.; Zhu, C.; Powis, G.; Kunkel, M. 3ß-Hydroxy-6-azacholestane and related analogues as phosphatidylinositol specific phospholipase C (PI-PLC) inhibitors with antitumor activity. Bioorg. Med. Chem. 2000, 8, 699-706.

(C) 2012 by the authors; licensee MDPI, Basel, Switzerland. This article is an open access article distributed under the terms and conditions of the Creative Commons Attribution license (http://creativecommons.org/licenses/by/3.0/). 